\title{
Estudio de la variabilidad espacial y temporal de los glaciares y ecosistemas locales del Volcán Antisana
}

\section{(Study of the spatial and temporal variability of local ecosystems and glaciers of the Antisana Volcano)}

\author{
Mauricio Valladares Borja ${ }^{1}$
}

\begin{abstract}
Resumen:
El interés científico sobre el cambio climático permitió descubrir evidencias que demuestran una tendencia de calentamiento general provocada por las actividades que desarrolla el ser humano. Hechos como el aumento del nivel del mar, eventos meteorológicos extremos y el retroceso de glaciares, constituyen indicadores de la presencia de modificaciones de los patrones climáticos normales. En el Ecuador, el volcán Antisana es un estratovolcán de importancia estratégica que influye en el clima de la región, en el funcionamiento de los ecosistemas locales y también constituye una reserva de agua para la población del Distrito Metropolitano de Quito (DMQ). Los glaciares de este volcán tropical también sufren los efectos del calentamiento global cuya disminución a lo largo del tiempo es motivo de preocupación y análisis. El presente trabajo de investigación consistió en realizar el estudio espacial y temporal basado en series históricas de fotografías aéreas obtenidas entre los años de 1956 y 2011, a través de las capacidades de geo-procesamiento de los sistemas de información geográfica (SIG). Los resultados obtenidos demuestran una disminución significativa de los glaciares del Volcán Antisana e importantes cambios en las formaciones ecológicas locales circundantes.
\end{abstract}

Palabras clave: Antisana; cambio climático; glaciares; fotografías; sig.

\begin{abstract}
:
The scientific interest in climate change allowed to uncover evidence demonstrating a general warming trend caused by human activities. Facts such as rising sea levels, extreme weather events and retreat of glaciers, are indicators of the presence of alterations of normal weather patterns. In Ecuador, the Antisana is a stratovolcano of strategic importance affecting the climate of the region, in the functioning of the surrounding ecosystems. It is also a reserve of water for the population of the Metropolitan District of Quito (DMQ). The glaciers of the tropical volcano are also suffering the effects of global warming. Its decline over time is of concern and should be analyzed. The present research work performed a spatial and temporal study based on historical series of aerial photographs taken between 1956 and 2011, through geoprocessing capabilities of geographic information systems (GIS). The results show a significant decrease in the glaciers of the Antisana Volcano and significant changes in the surrounding local ecological formations.
\end{abstract}

Keywords: Antisana; climate change; glaciers; photographs; gis.

\section{Introducción}

Existen evidencias claras de un cambio climático generalizado que representa una amenaza para la sociedad. Las principales pruebas de la modificación de los escenarios climáticos mundiales se manifiestan como aumento del nivel del mar, aumento de la temperatura global, calentamiento de

\footnotetext{
${ }^{1}$ Universidad Tecnológica Equinoccial, Facultad de Ciencias de la Ingeniería, Carrera de Ingeniería
} Ambiental y Manejo de Riesgos Naturales, Quito - Ecuador (hugo.valladares@ute.edu.ec) 
los océanos, disminución del hielo Ártico y Antártico, eventos meteorológicos extremos, acidificación de los océanos y retroceso de glaciares. El retroceso o disminución de los glaciares no se debe a un fenómeno estacional cíclico, ya que el glaciar no recupera su volumen inicial en los meses fríos, sino que año tras año va disminuyendo su volumen, lo que a su vez ocasiona alteraciones irreversibles en los ecosistemas locales.

Los Andes recorren el occidente de América del Sur en dirección norte-sur a lo largo de 7000 kilómetros y con un ancho que varía entre 200 a 700 kilómetros (García, 2013). Esta alineación montañosa surgida por la subducción de la Placa de Nazca bajo la Placa Sudamericana, se compone de numerosas cordilleras que en algunos casos alcanzan altitudes de más de 6000 metros sobre el nivel del mar (García, 2013). El Volcán Antisana es un estratovolcán localizado en la cordillera de Los Andes, situado entre las provincias de Napo y Pichincha, cuya altitud es de 5753 metros sobre el nivel del mar. Tiene una importancia singular en la región ya que influye en el clima, en el equilibrio de los ecosistemas locales y representa una reserva de agua para la población del Distrito Metropolitano de Quito (DMQ). En la Figura 1 se indica la ubicación del Volcán Antisana respecto a América de Sur y la ciudad de Quito.

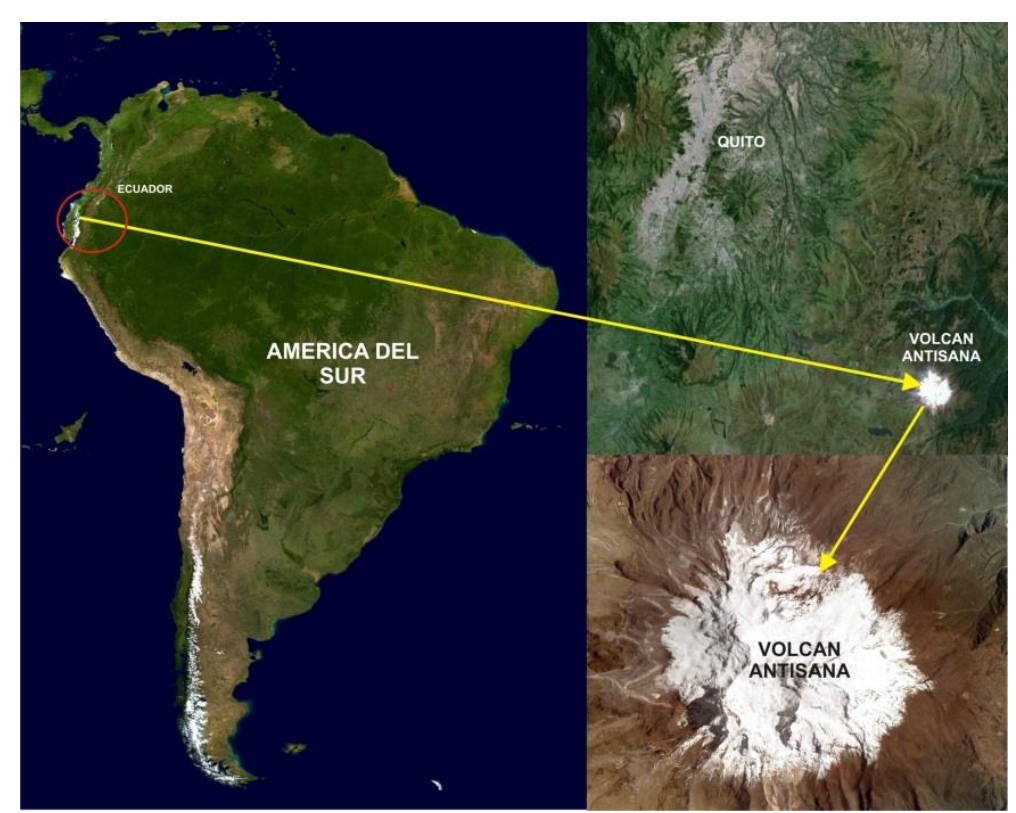

Figura 1. Ubicación del Volcán Antisana.

La localización de los glaciares tropicales puede determinarse mediante tres parámetros: 1. Trópicos astronómicos (limitación por radiación), 2. Áreas donde la amplitud térmica diaria supera la amplitud térmica anual (limitación térmica) y 3. La banda de oscilación de la Zona de Convergencia Intertropical o ITCZ, por sus siglas en inglés de Inter Tropical Convergence Zone, (limitación hídrica) (Kaser, 1995, 1998; Kaser, 1996). Según estos criterios se pueden identificar el Volcán Antisana como un glaciar tropical ya que se encuentra en un espacio en el que confluyen los tres criterios. En la Figura 2 se indica la delimitación de glaciares tropicales. 


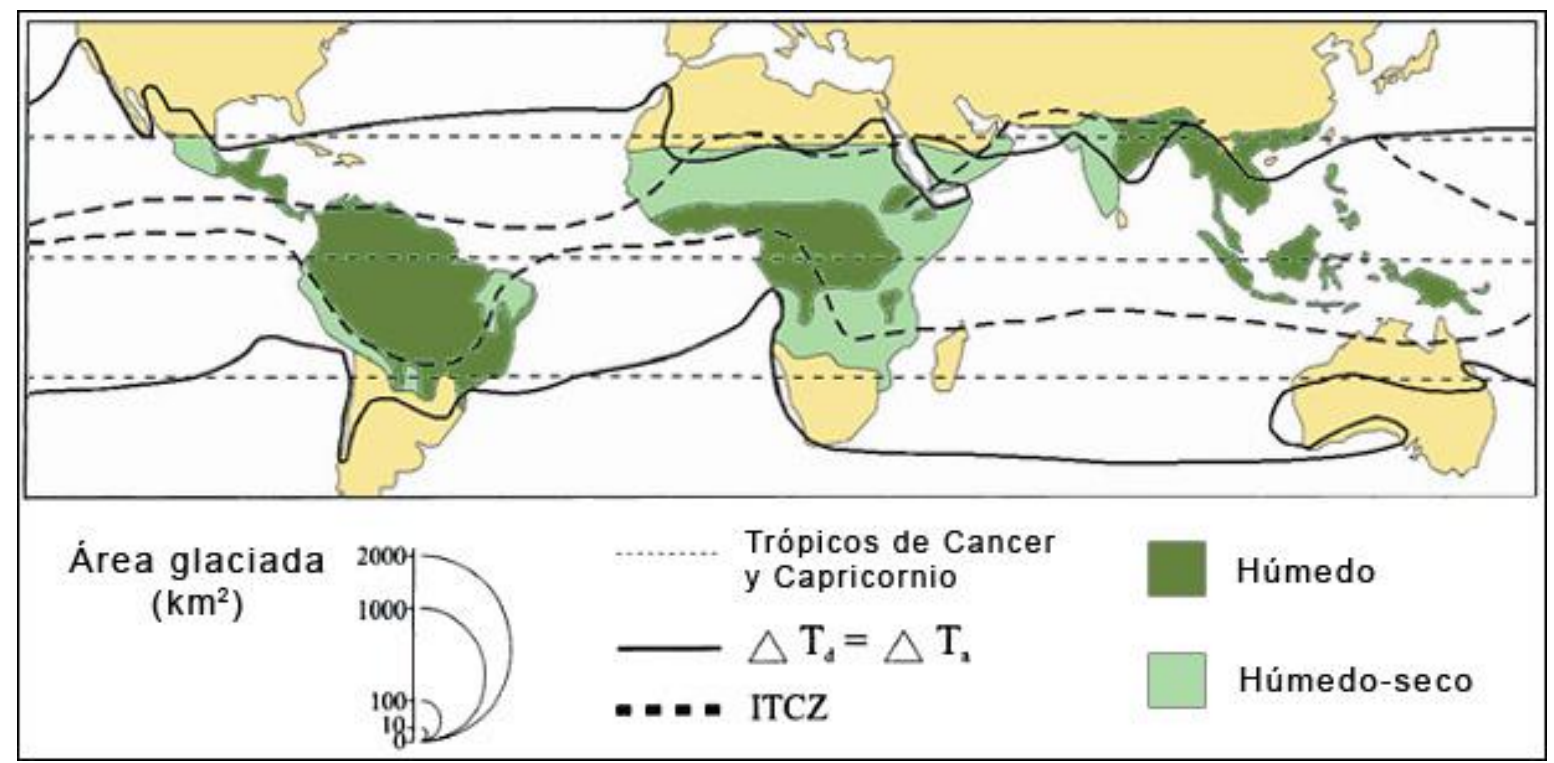

Figura 2. Ubicación del límite de glaciares tropicales.

El retroceso de los glaciares del Volcán Antisana ha motivado el interés de varios organismos nacionales e internacionales (Jordan, 1999; Francou, 2000; Cáceres, 2002; Cáceres, 2006; Cáceres, 2008) por tratar de entender la dinámica del glaciar y establecer medidas de adaptación oportunas frente a los efectos de este fenómeno. Este hecho determinó que en noviembre del año 2008 la Empresa Pública Metropolitana de Agua Potable y Saneamiento (EPMAPS) y el Instituto Geográfico Militar (IGM) suscribieran un convenio de cooperación que permitió obtener dos series de fotografías aéreas destinadas para el estudio de los glaciares del Volcán Antisana (Valladares, 2012), particularmente de los años 2009 y 2011, las mismas que conjuntamente con las fotografías aéreas disponibles desde el año 1956 constituyen los insumos básicos utilizados para esta investigación.

Considerando lo anterior, el propósito del presente trabajo consistió en realizar el análisis espacial y temporal del retroceso de los glaciares del Volcán Antisana y los ecosistemas locales a partir de las series de fotografías aéreas obtenidas entre los años 1956 y 2011, apoyado por las funciones de análisis y procesamiento de datos que incorporan los sistemas de información geográfica (SIG). En la Figura 3 mediante una línea de color rojo se observa el límite del área de estudio, la misma que incluye los glaciares del Volcán Antisana y las formaciones naturales circundantes (Valladares, 2012). 


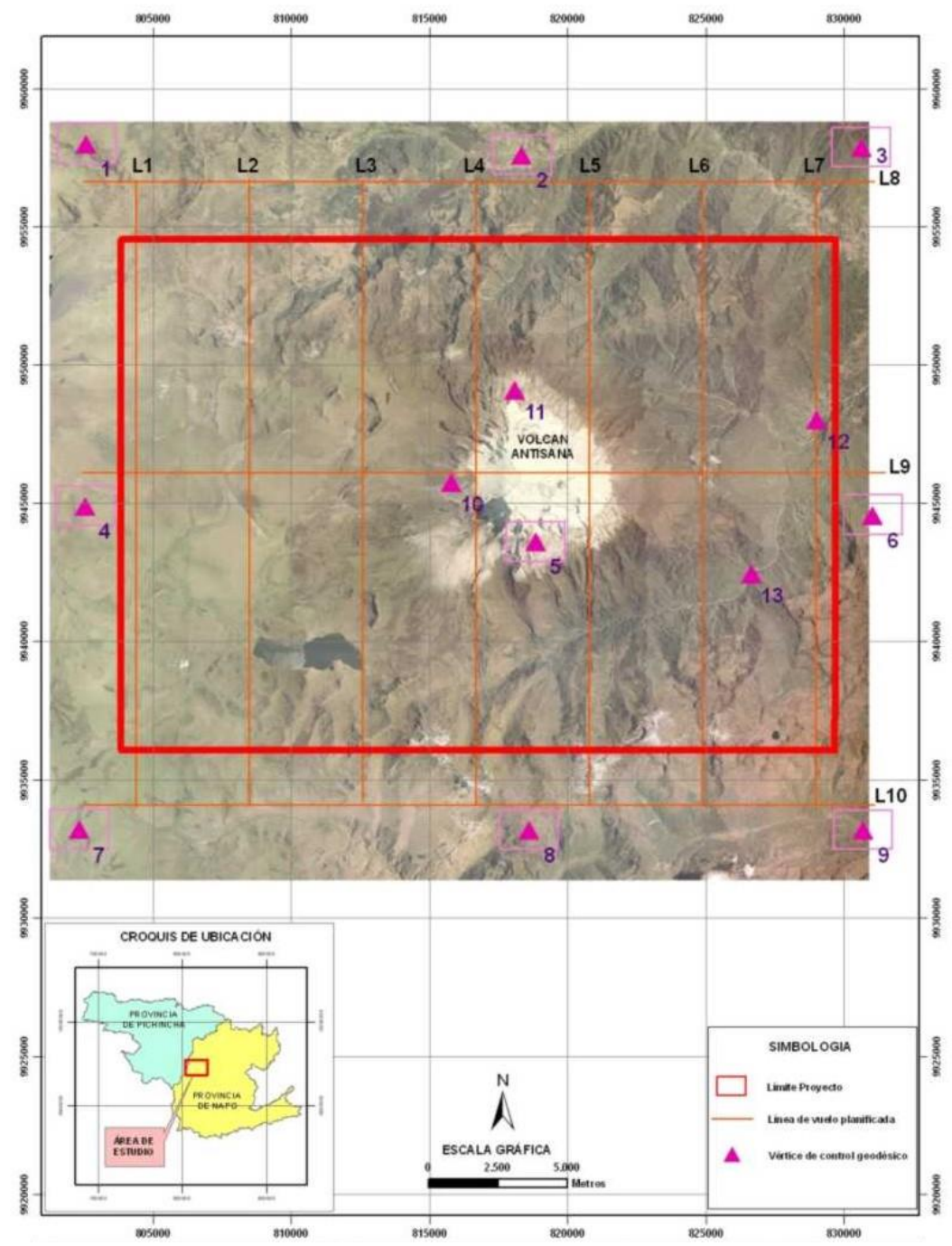

Figura 3. Ubicación del área de estudio.

\section{Metodología}

Debido a las complejidades del área de estudio y las relaciones existentes entre los glaciares del Volcán Antisana y las formaciones ecológicas locales, esta investigación utilizó el método heurístico (Bransford \& Stein, 1987) que se fundamenta en el análisis de los procesos físicos que actúan en el área de estudio, el mapeo de las coberturas glaciares de cada una de las series de datos, el mapeo de las diferentes formaciones ecológicas presentes en cada serie de fotografías y el estudio comparativo de las capas de información generadas.

Esta sección trata los procesos, técnicas y materiales empleados en la elaboración del trabajo y los distintos análisis que en él se recogen. En la Figura 4 se describe a través de un diagrama de flujo las fases del procedimiento metodológico utilizado, el mismo que garantiza la exactitud de la información espacial georeferenciada que fundamenta la ulterior fase de interpretación y deducción de resultados. 
Toma de fotografía aérea nueva específica

Fotografías aéreas escala 1:30.000 de los glaciares del

Volcán Antisana en formato analógico (2009 y 2011)
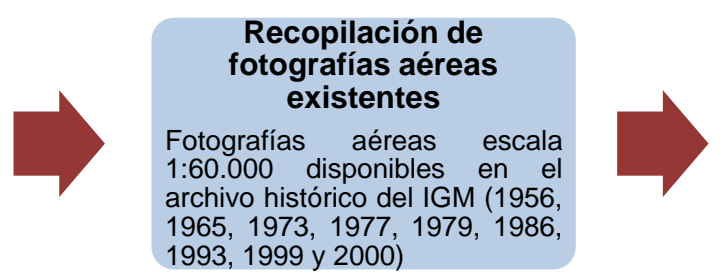

Escaneo fotogramétrico

Escaneo de las películas fotográficas mediante un escáner fotogramétrico digital a una resolución espacial de 14 um y una resolución radiométrica de 24 bits

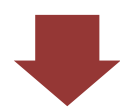

Control de campo vertical Nivelación geométrica, nivelación trigonométrica modelo geopotencial EGM96

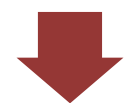

Fotogrametría digital Orientación y ajuste de bloques de fotografías aéreas. Restitución fotogramétrica escala 1:25.000. Generación de modelos digitales del terreno (dtm). Creación de ortofotos

\section{Control de campo} horizontal
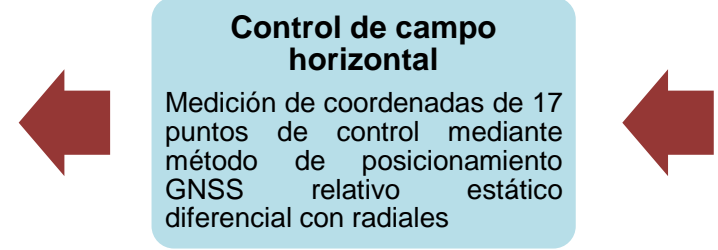

Evaluación fotogramétrica Análisis del cubrimiento estereoscópico de las fotografías aéreas. Se determinó un recubrimiento longitudinal del $70 \% \pm 20 \%$ y un recubrimiento lateral del $40 \% \pm$ $20 \%$

Figura 4. Diagrama de flujo del procedimiento metodológico desarrollado.

\section{Obtención y procesamiento de las fotografías aéreas de los años 2009 y 2011}

La ejecución del convenio suscrito entre la EPMAPS y el IGM ofrece la posibilidad de contar con insumos técnicos específicos destinados al estudio de los páramos y glaciares del Volcán Antisana (Valladares, 2012). En ese contexto en septiembre del año 2009 y octubre del año 2011 se obtuvieron sendas fotografías aéreas destinadas para la investigación del Volcán Antisana. Para cubrir el área de estudio cada serie requirió 10 líneas de vuelo y 149 fotografías aéreas en escala 1:30.000, las mismas que fueron sometidas a un proceso de evaluación aerofotogramétrica que determinó que la calidad de las imágenes es óptima, es decir no existe presencia de sombras, nubes, ni bruma, y que la definición de las imágenes y el contraste son adecuados. A continuación se desarrolló la fase de escaneo de la película fotográfica mediante un escáner fotogramétrico digital de una resolución espacial de $14 \mu \mathrm{m}$ y una resolución radiométrica de 24 bits. El resultado de esta primera fase consistió en dos series de fotografías aéreas a una escala de 1:30 $000 \pm$ $15 \%$, con un recubrimiento longitudinal del $70 \% \pm 20 \%$ y un recubrimiento lateral del $40 \% \pm 20 \%$ que permiten un adecuado cubrimiento estereoscópico de la zona de estudio y se consideran 
aptas para fines cartográficos. Cabe indicar que para obtener estas fotografías se empleó el avión Cessna Citation II IGM-628, la cámara fotográfica RC-30 y película a color (Valladares, 2012).

\section{Obtención y procesamiento de las fotografías aéreas del archivo histórico}

Para establecer una serie de datos representativa que sustente de manera sólida la investigación de la variabilidad espacial y temporal del área de interés se obtuvieron las fotografías aéreas de los siguientes años: 1956 (Hastenrath 1981; Cáceres 2003; Cáceres, 2004; Cáceres 2005; Cáceres 2008; Jordan, 2003; Sauer 1965), 1965, 1973, 1977, 1979, 1986, 1993, 1999 у 2000 disponibles en el archivo histórico del IGM. Luego de la evaluación fotogramétrica de los datos obtenidos se concluyó que las series de fotografías válidas para el estudio corresponden a 5 épocas: 1956, 1965, 1977-1979, 1993 y 1999-2000 (Valladares, 2012). Además al tratarse de fotografías aéreas históricas destinadas a otro fin distinto que el estudio de glaciares la escala es más pequeña y tiene un valor aproximado de 1:60.000. Las fotografías que superaron el proceso de evaluación se consideran aptas para fines cartográficos y fueron digitalizadas mediante un escáner fotogramétrico digital cuya resolución espacial y radiométrica es la misma que se empleó para escanear las fotografías de los años 2009 y 2011.

Como resultado de estas dos primeras fases de obtención y procesamiento de datos se obtuvieron siete series de fotografías aéreas (1956, 1965, 1977-1979, 1993, 1999-2000, 2009 y 2011) que describen la evolución del Volcán Antisana durante 55 años, es decir más de medio siglo de historia.

\section{Trabajos de campo de control horizontal}

Este trabajo consistió en determinar las coordenadas planas (Este y Norte) de 17 puntos de control distribuidos en 9 puntos de apoyo fotogramétrico y 8 puntos de apoyo topográfico. Los puntos de apoyo fotogramétrico se distribuyeron de manera uniforme en el bloque de fotografías aéreas y permitieron ajustar las fotografías aéreas. Por otra parte los puntos topográficos y sus respectivas marcas azimutales se ubicaron sobre todo en el sector nororiental del Volcán Antisana y se emplearon en varios trabajos de topografía de detalle planificados por la EPMAPS. EI control de campo horizontal se realizó mediante el método de posicionamiento GNSS relativo estático diferencial con radiales. El tiempo de medición en los puntos de apoyo tuvo una duración mínima de una hora y máximo de dos horas, con intervalos de grabación de 5 segundos. Para realizar este trabajo se utilizaron receptores geodésicos de doble frecuencia $(L 1, L 2)$, código $C / A$ (Coarse/Adquisition) y el código $\mathrm{P}$ (Precise). El establecimiento del control horizontal partió de vértices de la red GPS del Distrito Metropolitano de Quito y de la red geodésica nacional del IGM. En la Figura 5 se muestran fotografías de los equipos utilizados para realizar los trabajos de control horizontal en el área de interés (Valladares, 2012). 

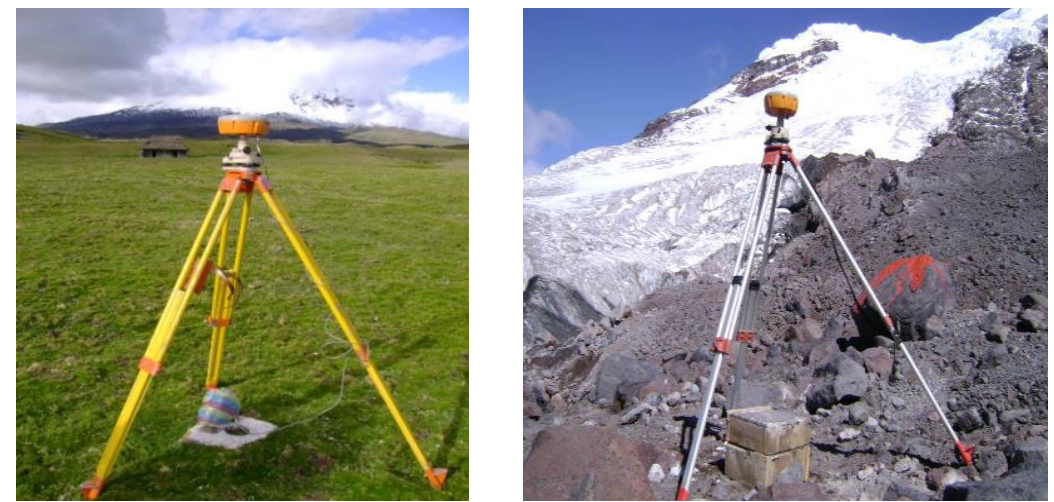

Figura 5. Trabajos de control horizontal realizados en la zona de estudio.

\section{Trabajos de campo de control vertical}

Para la determinación de las alturas sobre el nivel medio del mar de los puntos de control se partió de vértices de control vertical de las redes geodésicas anteriormente citadas, y se empleó nivelación geométrica, trigonométrica o el modelo geopotencial EGM96. Respecto al método de nivelación se debe indicar que un punto (equivalente al 5,88\%) se niveló geométricamente, 5 puntos (equivalente al $29,41 \%$ ) tienen nivelación trigonométrica y 11 puntos (equivalente al $64,71 \%)$ tienen nivelación ortométrica. El tipo de nivelación utilizada dependió exclusivamente del grado de dificultad de acceso a los sitios de trabajo por lo que los porcentajes indicados demuestran la complejidad de los trabajos de campo (Valladares, 2012).

Luego del post procesamiento de la información se determinó que las diferencias encontradas en los vértices de control horizontal se encontraban en el orden de 0,013 m en Norte (DN) y de 0,050 $m$ en Este (DE), mientras que los errores de cierre de los trabajos de nivelación se encontraron en el orden de 0,005 m (DZ) (Valladares, 2012).

Respecto al sistema de referencia espacial, la medición se realizó en el sistema de referencia WGS84 (Sistema Geodésico Mundial de 1984) y en la proyección cartográfica UTM (Universal Transversa de Mercator) y luego las coordenadas fueron transformadas al sistema de referencia espacial del Distrito Metropolitano de Quito (WGS84 y Transversa de Mercator Modificada) (Valladares, 2012).

Los puntos de control medidos en campo fueron marcados (o pinchados) en las respectivas fotografías aéreas, de tal forma de facilitar la fotoidentificación de dichos puntos y poder continuar con la siguiente etapa de fotogrametría digital (Valladares, 2012).

\section{Trabajos fotogramétricos}

El trabajo de fotogrametría digital consistió en realizar medidas exactas sobre las fotografías aéreas recopiladas y preparadas anteriormente. Para ello se emplearon los listados de coordenadas y alturas obtenidos en los trabajos de campo horizontal y vertical. En esta fase se realizó la restitución fotogramétrica digital en escala 1:25.000 conformada principalmente por 
curvas de nivel (altimetría) y quebradas (hidrografía) existentes en la zona de interés. A partir de la altimetría e hidrografía extraídas de las fotografías aéreas se crearon los modelos digitales del terreno (DTM) en formato GeoTiff y posteriormente las ortofotografías u ortofotos (Valladares, 2012).

Para la elaboración de las ortofotos se utilizó el algoritmo de rectificación de "nearest neighbor" (vecino más cercano) del programa Orthopro que minimiza los errores provocados por corrimientos y distorsiones radiométricas. Finalmente, como resultado de esta fase se obtuvieron las ortofotos y el ortofotomosaico del área de estudio en cada época estudiada. En la Figura 6 se ilustran modelos digitales del terreno generados en formato GeoTiff (Valladares, 2012), y en la Figura 7 se ilustran perspectivas de ortofotos sobrepuestas en sus respectivos modelos digitales del terreno (Valladares, 2012).
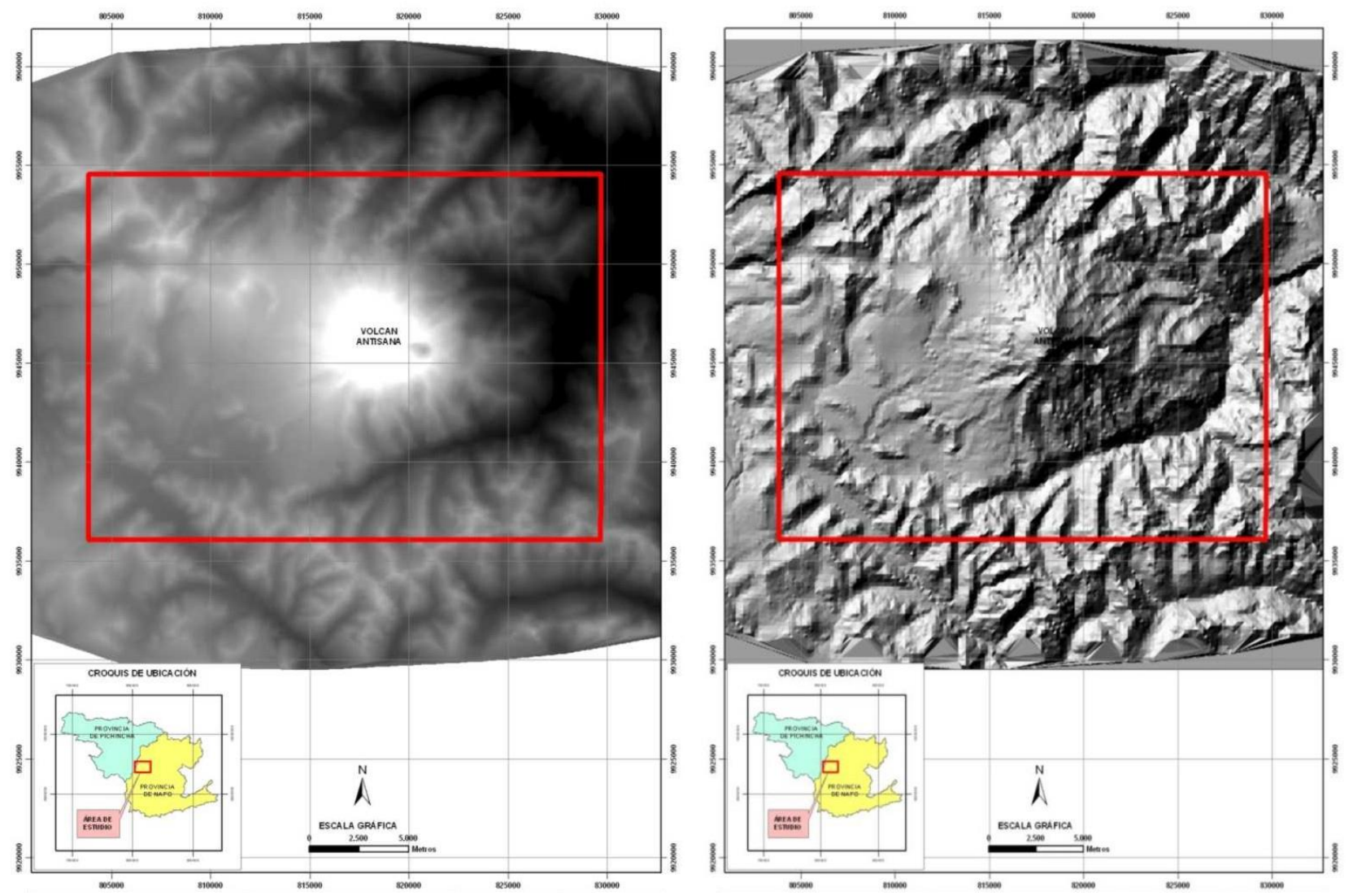

Figuras 6. Ejemplos de modelos digitales del terreno obtenidos a partir de fotografías del año 2009. 

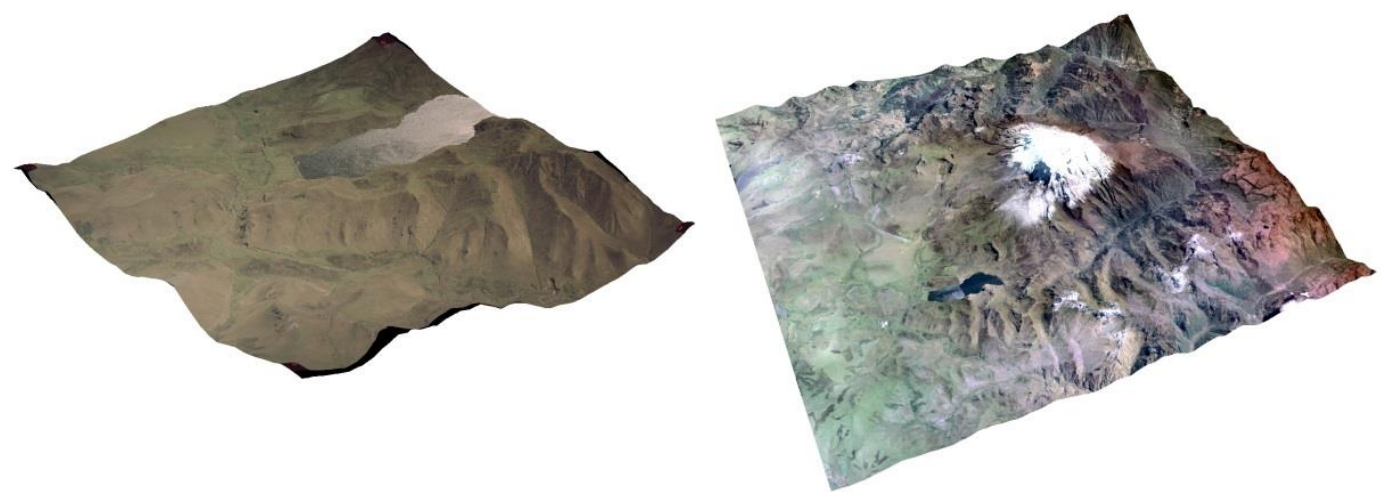

Figura 7. Ejemplos de perspectivas de ortofotos sobrepuestas en sus respectivos modelos digitales del terreno (Año 2009).

En la siguiente Figura 8 se indican las siete series de fotografías aéreas generadas que describen la evolución del Volcán Antisana durante 55 años, desde el año 1956 hasta el año 2011. Allí las cinco primeras series (1956, 1965, 1977-1979, 1993, 1999-2000) están conformadas por fotografías aéreas escala 1:60.000 en blanco y negro obtenidas del archivo histórico nacional, mientras que las dos últimas series de datos (2009 y 2011) corresponden a fotografías aéreas nuevas en escala 1:30.000 a color que facilitan la foto identificación de los glaciares y otras formaciones naturales locales (Valladares, 2012).

\section{Estimación fotogramétrica de la cobertura glaciar}

La determinación del límite de la cobertura glaciar de cada serie se realizó mediante la fotoidentificación de formaciones de hielo y en aquellos casos donde la nieve dificultó la discriminación de la masa de hielo se realizó una comparación estereoscópica. A continuación se efectuó la sustracción de modelos digitales del terreno de los años 1956 y 2011 obteniendo así un modelo de variación de espesor. Mediante este procedimiento de cálculo se determinó que la reducción de altitud provocada por la fusión en el glaciar durante el período 1956 - 2011 fue de 30,05 m (Valladares, 2012). A continuación se efectuó la estimación del volumen de hielo de glaciar como el producto de la superficie glaciar medida sobre la ortofoto del año 2011 por la altura media obtenida mediante el cálculo de la variación de espesor. De esta forma se determinó que la variación de volumen de hielo acumulado está en el orden de 535'965 233,20 m³, que expresado en volumen de agua representa 482'368 709,88 $\mathrm{m}^{3}$. En tanto que el caudal medio equivalente para un período de 55 años (1956-2011) es de 288,55 l/s (Valladares, 2012).

En síntesis, para una superficie total del glaciar de 17'835 781,47 m² (ortofoto 2011) la variación media del espesor en un período de 55 años (1956-2011) es de aproximadamente 30,05 m, con una pérdida aproximada de volumen de hielo de 482'368 709,88 $\mathrm{m}^{3}$, que equivale a un caudal medio de 288,55 l/s (Valladares, 2012).

En la Figura 9 se ilustra la delimitación de los glaciares del Volcán Antisana sobre la ortofoto del año 2011. 


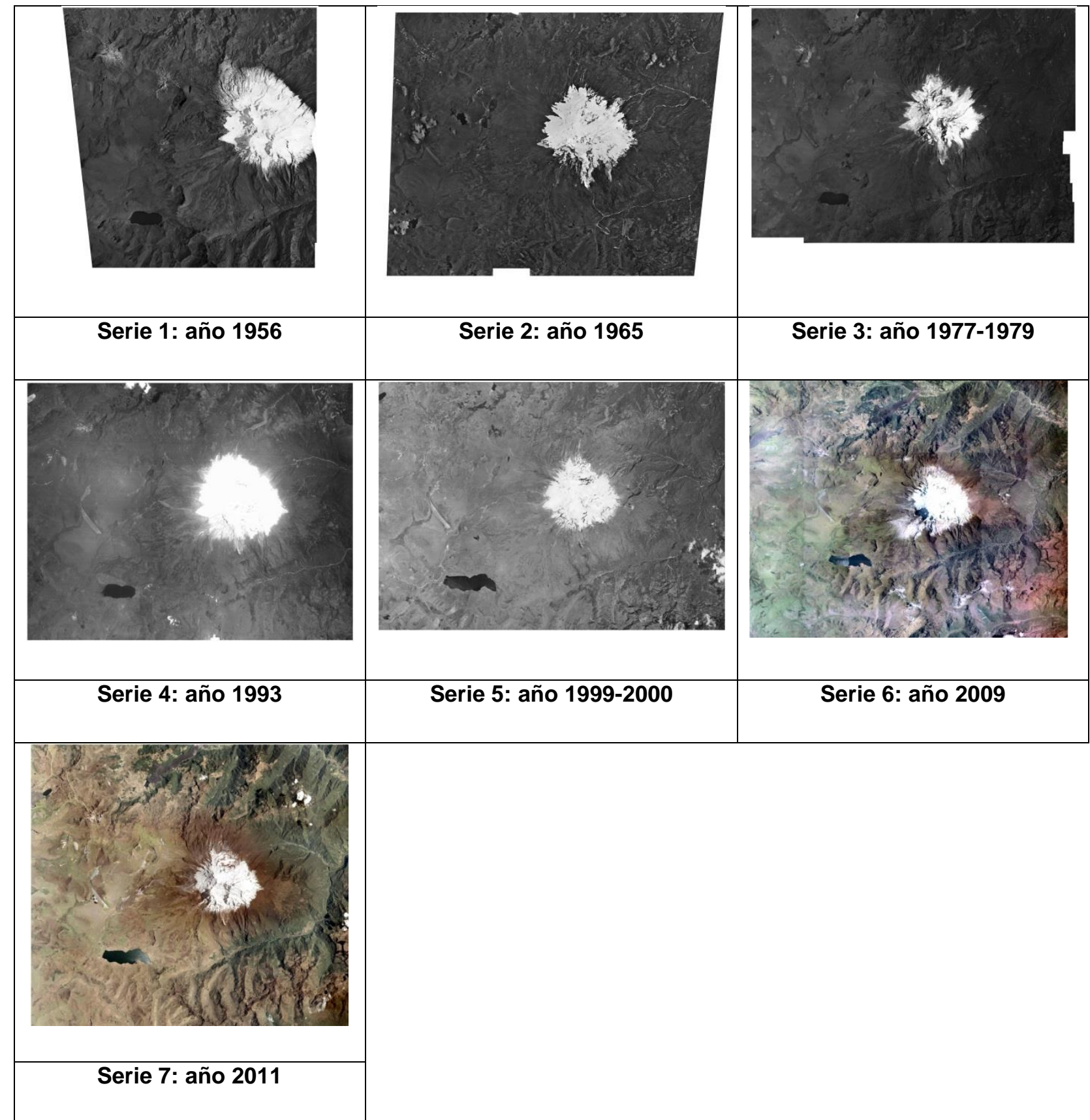

Figura 8. Series de datos de fotografías aéreas.
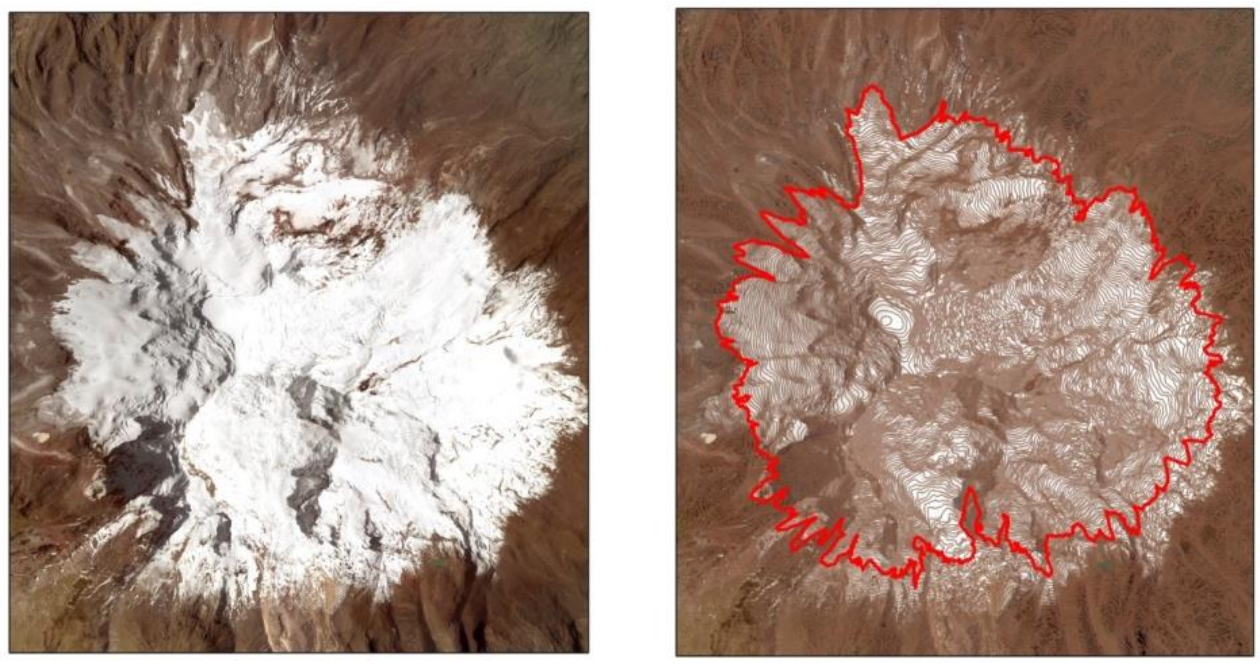

Figura 9. Límite glaciar del año 2011. 


\section{Cambios en las formaciones naturales del área de estudio}

La determinación de los cambios producidos en las formaciones ecológicas locales requirió de un minucioso trabajo de fotointerpretación de cada una de las series de fotografías aéreas recopiladas. Las diferencias encontradas entre las unidades temáticas de las diversas épocas interpretadas constituyen una evidencia del cambio sufrido en las formaciones naturales.

Las principales formaciones naturales encontradas corresponden a cuerpos de agua, áreas erosionadas, bosque natural, bosque plantado, lava, glaciar, páramo, pasto cultivado, pasto natural, vegetación arbustiva baja, arenales y cultivos de ciclo corto (Valladares, 2012). La comparación temporal de las formaciones ecológicas indica la presencia de un intenso proceso erosivo, seguramente debido a las fuertes corrientes de viento que soplan en la región y al intenso pastoreo de ganado bovino existente.

Otra evidencia de los importantes cambios ocurridos en la zona se ilustra en la Figura 10. Allí se muestra la variación espacial de la Laguna La Mica en un período de 55 años. El espejo de agua de la Laguna La Mica alcanzaba una extensión de $1,90 \mathrm{Km}^{2}$ en el año 1956 y luego de la construcción del Proyecto La Mica Quito Sur en el año 2000 la superficie inundada se incrementó en un $175,26 \%$ (Valladares, 2012).
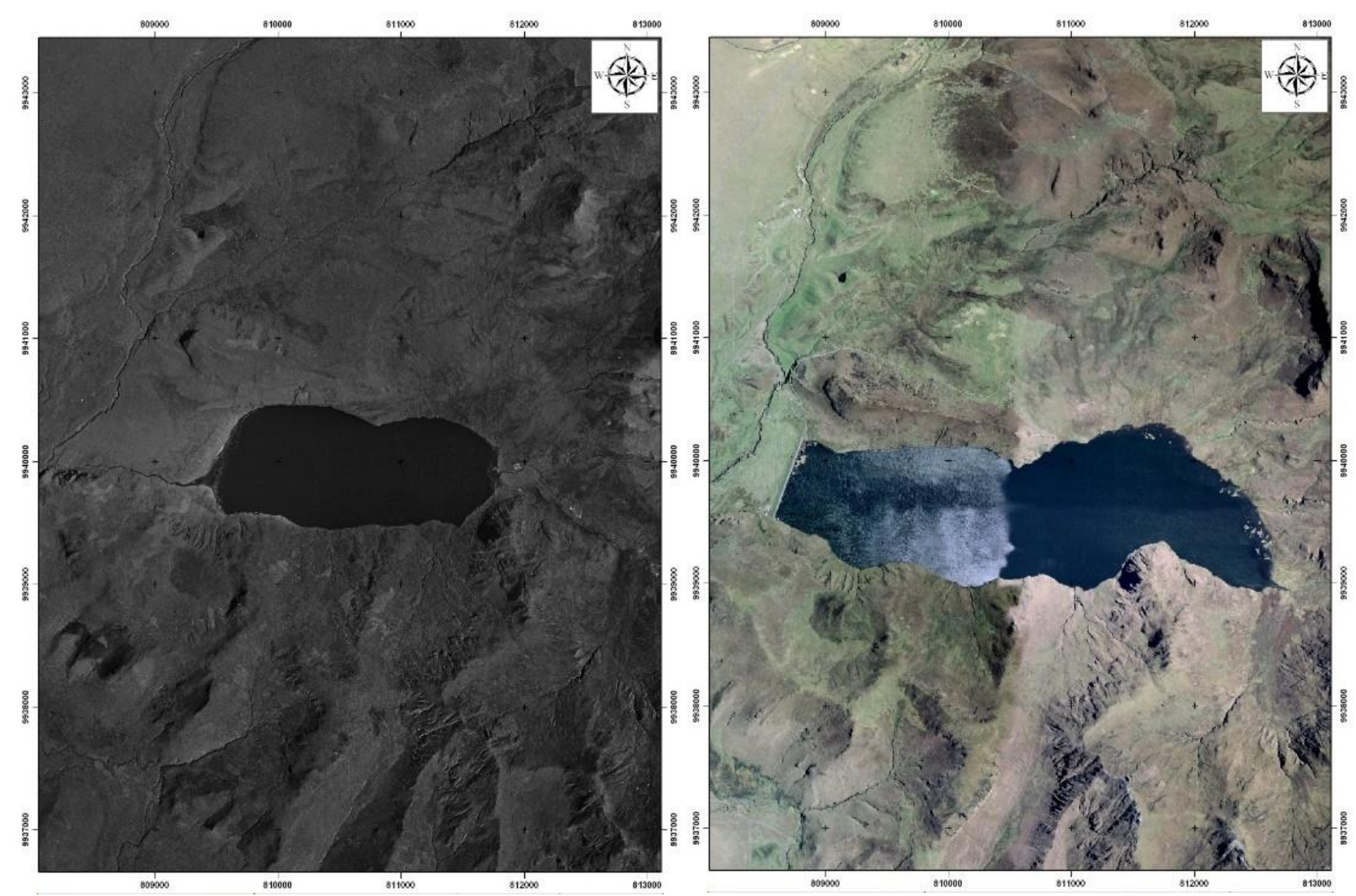

Figura 10. Fotografía de la Laguna La Mica en el año 1956 (izquierda) y fotografía del Embalse La Mica en el año 2011 (derecha). 
En la Figura 11 se muestran los mapas de uso del suelo y cobertura vegetal obtenidos a partir de las fotografías aéreas de los años 1956 y 2011, los mismos que fueron elaborados en el sistema de información geográfico ArcGIS.
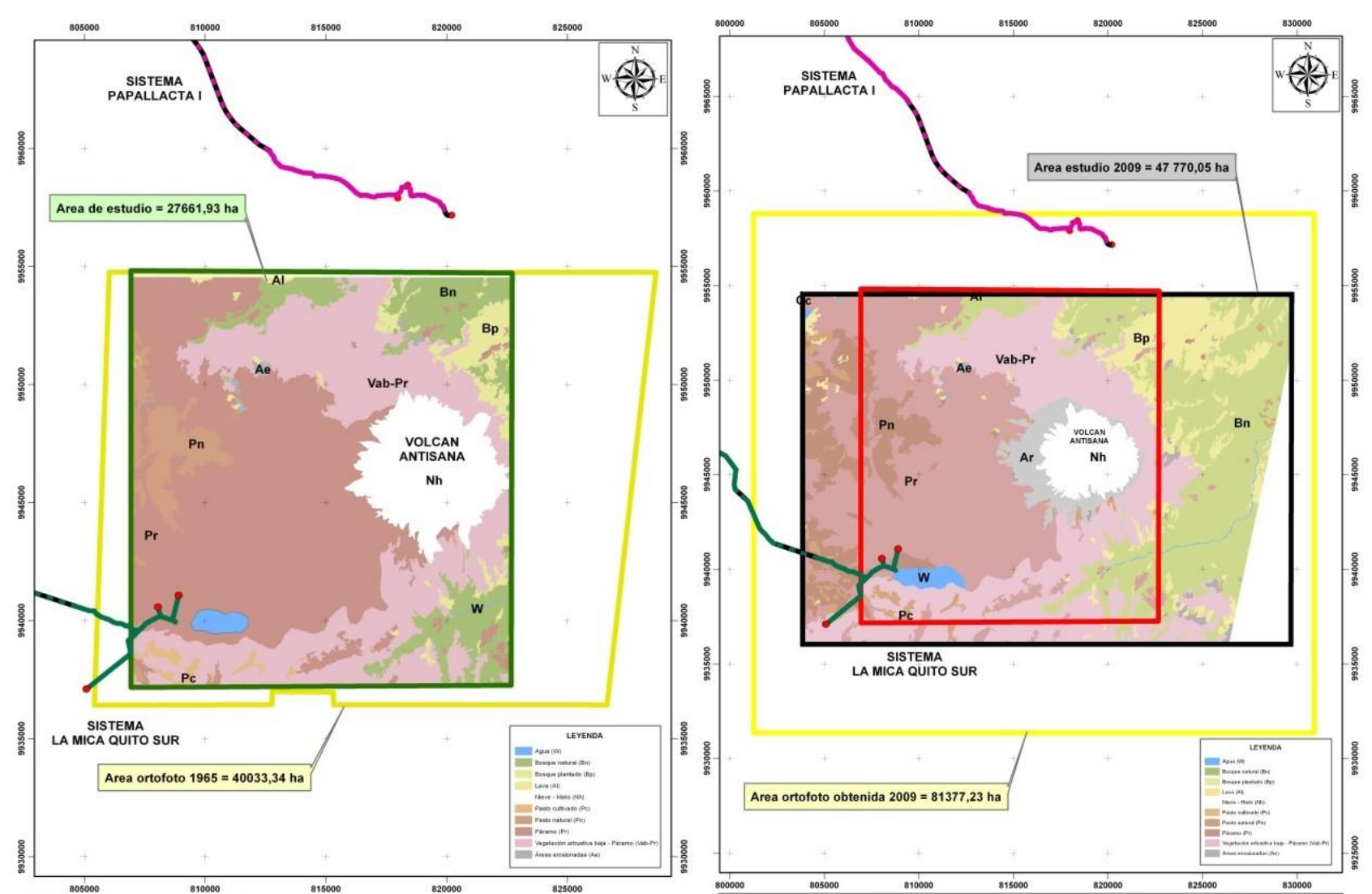

Figura 11. Mapas de uso del suelo y cobertura vegetal obtenidos a partir de las fotografías de 1956 y 2011.

\section{Resultados}

El presente trabajo realizó la recopilación y procesamiento de 7 series de fotografías aéreas correspondientes a los años: 1956, 1965, 1977-1979, 1993, 1999-2000, 2009 y 2011 (Valladares, 2012) que representan la evolución histórica del Volcán Antisana durante un período de 55 años y simulan la dinámica glaciar y los cambios ocurridos en las formaciones ecológicas circundantes. Este proceso fue apoyado con los respectivos trabajos de escaneo, evaluación fotogramétrica, control horizontal, control vertical y fotogrametría digital.

A partir de la altimetría e hidrografía extraídas de las fotografías aéreas se generaron los modelos digitales del terreno. La sustracción de los modelos digitales del terreno de los años 1956 y 2011 permitió obtener un modelo de variación de espesor. Mediante este procedimiento de cálculo se determinó que la reducción de altitud provocada por la fusión en la zona glaciar en el período comprendido entre 1956 y 2011 fue de 30,05 m (Valladares, 2012).

En la Tabla 1 se muestran las superficies glaciares extraídas de cada una de las series de datos de fotografías aéreas. Allí se puede observar que la superficie glaciar registrada en el año 1956 era $35,380 \mathrm{Km}^{2}$ y que la superficie identificada en el año 2011 era $17,515 \mathrm{Km}^{2}$, comprobándose un 
importante retroceso en los glaciares del Volcán Antisana. Los datos también nos sugieren que las dos últimas series, 2009 y 2011, no tienen una separación temporal suficiente para encontrar cambios significativos en los glaciares estudiados.

Tabla 1. Variación de la superficie glaciar durante 55 años (1956-2011).

\begin{tabular}{|c|c|c|c|}
\hline No. & Año & $\begin{array}{c}\text { Superficie glaciar } \\
\left(\mathbf{K m}^{\mathbf{2}}\right)\end{array}$ & $\begin{array}{c}\text { Variación superficie } \\
\text { glaciar }\left(\mathbf{K m}^{2} \mathbf{)}\right.\end{array}$ \\
\hline 1 & 1956 & $35,380\left(^{\star}\right)$ & - \\
\hline 2 & 1965 & 27,721 & 7,659 \\
\hline 3 & $1977-1979$ & 24,466 & 3,255 \\
\hline 4 & 1993 & 25,915 & 1,449 \\
\hline 5 & $1999-2000$ & 20,063 & 5,852 \\
\hline 6 & 2009 & 17,836 & 2,227 \\
\hline 7 & 2011 & 17,515 & 0,321 \\
\hline
\end{tabular}

$\left(^{\star}\right)$ : La antigüedad de las imágenes no facilita una adecuada discriminación del glaciar

La Tabla 2 indica las principales formaciones ecológicas locales encontradas en el proceso de interpretación de las series de datos de fotografías aéreas. Estas formaciones naturales corresponden a cuerpos de agua, áreas erosionadas, bosque natural, bosque plantado, lava, glaciar, páramo, pasto cultivado, pasto natural, vegetación arbustiva baja, arenales y cultivos de ciclo corto (Valladares, 2012). La comparación temporal de las formaciones ecológicas indica la presencia de un intenso proceso erosivo, seguramente debido a las fuertes corrientes de viento que soplan en la región y al intenso pastoreo de ganado bovino existente.

Tabla 2. Variación de las superficies de las formaciones naturales locales durante 55 años (1956-2011).

\begin{tabular}{|c|c|c|c|c|c|c|c|c|}
\hline \multirow[t]{2}{*}{ No. } & \multirow{2}{*}{$\begin{array}{c}\text { Formación } \\
\text { natural }\end{array}$} & \multicolumn{7}{|c|}{ Superficie $\left(\mathrm{Km}^{2}\right)$} \\
\hline & & 1956 & 1965 & 1977-1979 & 1993 & 1999-2000 & 2009 & 2011 \\
\hline 1 & Agua & 0,115 & 0,115 & 0,115 & 0,115 & 4,097 & 4,097 & 4,097 \\
\hline 2 & Áreas erosionadas & 1,297 & 1,297 & 1,297 & 1,297 & 1,297 & 3,536 & 3,536 \\
\hline 3 & Bosque natural & 27,002 & 27,002 & 27,002 & 27,002 & 27,002 & 94,415 & 94,415 \\
\hline 4 & Bosque plantado & 10,742 & 10,742 & 10,742 & 10,742 & 10,742 & 35,349 & 35,349 \\
\hline 5 & Lava & 0,300 & 0,300 & 0,300 & 0,300 & 0,300 & 0,300 & 0,300 \\
\hline 6 & Glaciar & 35,380 & 27,721 & 24,466 & 25,914 & 20,062 & 17,836 & 17,515 \\
\hline 7 & Páramo & 102,264 & 109,923 & 113,179 & 111,730 & 113,600 & 120,540 & 120,219 \\
\hline 8 & Pasto cultivado & 2,362 & 2,362 & 2,362 & 2,362 & 2,362 & 9,463 & 9,463 \\
\hline 9 & Pasto natural & 12,121 & 12,121 & 12,121 & 12,121 & 12,121 & 35,389 & 35,389 \\
\hline 10 & $\begin{array}{l}\text { Vegetación arbustiva } \\
\text { baja }\end{array}$ & 81,452 & 81,452 & 81,452 & 81,452 & 81,452 & 111,264 & 111,264 \\
\hline 11 & Arenales & & & & & & 19,872 & 19,872 \\
\hline 12 & Cultivos ciclo corto & & & & & & 0,037 & 0,037 \\
\hline & Total $\left(\mathrm{Km}^{2}\right)$ & 273,037 & 273,037 & 273,037 & 273,037 & 273,037 & 452,103 & 452,103 \\
\hline
\end{tabular}

\section{Conclusiones y Recomendaciones}

Los datos obtenidos evidencian la necesidad de que el país implemente y mantenga programas técnicos sostenidos de monitoreo de glaciares tropicales, los mismos que permitan generar información de base necesaria para comprender de mejor forma la dinámica glaciar y los cambios que podrían esperarse en los ecosistemas locales relacionados con los glaciares. 
Varias predicciones indican que el cambio climático es capaz de modificar la disponibilidad y las necesidades de agua en todo el planeta; por ello el caso del Volcán Antisana es importante y requiere continuar con trabajos de investigación orientados a determinar el aporte real de los glaciares al sistema de abastecimiento de agua de la ciudad de Quito.

En una superficie total del glaciar de $17,515 \mathrm{Km}^{2}$ (ortofoto 2011) la variación media del espesor en un período de 55 años (1956-2011) es de aproximadamente 30,05 m, que representa una pérdida aproximada de volumen de hielo de 482' 368 709,88 $\mathrm{m}^{3}$ equivalente a un caudal medio de 288,55 I/s. Este caudal representa el 3,5\% de la demanda bruta de $8300 \mathrm{l} / \mathrm{s}$ requerida por la población total del DMQ calculada actualmente en 2' 418046 habitantes.

En relación con el estudio comparativo de las formaciones ecológicas circundantes a los glaciares del Volcán Antisana, éste permitió identificar las siguientes formaciones naturales: cuerpos de agua, áreas erosionadas, bosque natural, bosque plantado, lava, glaciar, páramo, pasto cultivado, pasto natural, vegetación arbustiva baja, arenales y cultivos de ciclo corto. La comparación temporal de dichas formaciones ecológicas indica la presencia de un intenso proceso erosivo, que está directamente relacionado con las fuertes corrientes de viento que soplan en la región y la presencia de ganado bovino que durante décadas ha pastado en la zona. Así mismo se determinó que dos ecosistemas naturales predominan en la zona: las formaciones de páramo y la vegetación arbustiva baja.

El agua dulce disponible, es decir la que podríamos aprovechar fácilmente queda limitada a sólo el 0,4 por ciento del total del agua presente en el planeta, lo que determina que este recurso es escaso o, cuando menos, limitado. Si a ello se suma el incremento de la población podríamos suponer una importante afectación en los sistemas de abastecimiento de agua locales. Esto implica modificar tendencias clave para alcanzar un aprovechamiento sostenible de los recursos hídricos como es el caso de limitar la expansión de la frontera agrícola y realizar una adecuada gestión de cuencas hidrográficas.

Las herramientas de procesamiento de información espacial georeferenciada que incorporan los sistemas de información geográfica resultan fundamentales para simular procesos de variabilidad espacial, tal como los ocurridos en los glaciares y ecosistemas circundantes del Volcán Antisana. Por ello cualquier tipo de investigación que involucre el análisis de una variable susceptible de ser representada espacialmente debe emplear necesariamente un sistema de información geográfica. 


\section{Bibliografía}

Bransford \& Stein. (1987). Solución ideal de problemas. Guía para mejor pensar, aprender y crear. Barcelona. Ed. Labor.

Cáceres, B. (2010). Actualización del Inventario de tres casquetes glaciares del Ecuador. Informe de Pasantía de Investigación en Université Nice Sophia Antipolis.

García, E. (2013). Evolución Glaciar del cuadrante noreste del Nevado Coropuna. Trabajo Final de Master. Universidad Complutense de Madrid.

Jordan E., 1983. Die Vergletscherung Des cotopaxi-ecuador. Zeitschrift Für Gletscherkunde und Glazialgeologie 9 (1), Universitätsverlag Wagner, Innsbruck, 73-102.

Jordan, E., 2007. Informe restitución fotogramétrica del volcán Cotopaxi DALE Digital. Inédito. Quito.

Jordan E., Blanco D.,Castillo, K.,Cruz, M.,González, J.,Torres, J.,Ungerechts, L.,Peñafiel, A.,Cáceres, B.,Francou, B.,.Primer ortofotomapa del Chimborazo (Ecuador) y su valor como diagnóstico para cambios climatológicos en relación con otros glaciares tropicales. VII Encuentro internacional de investigadores del grupo de trabajo de nieves y hielos de América Latina del PHI-UNESCO. Bogota 2008.

Jordan, E., Cáceres, B., Francou, B., Ungerechts, L., 2003. Die Glazialforschungen Hans Meyers aus heutiger Sicht Wertung der wissenschaftlichen Leistungen Meyers in den Hochanden von Ekuador aus aktueller Sicht und Ausblick auf die gegraphischen Forschungsergebnisse der vergangen 100 Jahre. Die Anden - Gegraphische Erforschung und künstlerische Darstellung. Wissenschaftliche Alpenvereinshefte 37, München 2003, S. 159-193.

Jordan, E., Hastenrath, S., 1999: Glaciers of Ecuador. In: Williams, R.S., Ferrigno, J.G., (Eds.), Satellite image atlas of the glaciers of the world - South America. USGS Professional paper 1386-I, 31-50.

Jordan, E., Ungerechts, L., Cáceres B., Francou B., Peñafiel A.. Francou B., 2005: Estimation by Photogrammetry of the glacier recession on the Cotopaxi Volcano (Ecuador) between 1956 and 1997. Hydrological Sciences Journal, 50 (6) December 2005, pp 949-961.

Kaser, G., 1995. Some notes on the behaviour of tropical glaciers. Bull. Inst. Fr. Etudes Andines 24 (3)., 671-681.75.

Kaser, G., 1996. Gletscher in den Tropen-ein Beitrag zur Geographie der tropischen Hochgebirge. Habilitationsschrift, Universit"at Innsbruck. 
Kaser, G., 1998. The Nature of Tropical Glaciers. International Hydrological Series.UNESCOCambridge Univ. Press (accepted and in preparation).English version of: Kaser, G.,1996. Gletscher in den Tropen-ein Beitrag zur Geographieder tropischen Hochgebirge. Habilitationsschrift, Universitat Innsbruck.

Kaser,G., 1999. A review of the modern fluctuations of tropical glaciers. Global and Planetary Change 22(1999) 93-103.

Kaser G., Georges C., 1997. Changes of the equilibrium-line altitude in the tropical Cordillera Blanca, Peru, 1930-50, and their spatial variations. Ann. Glaciol. 24 (1997) 344-349.

Kaser, G., Georges, C., 1999: On the mass balance of low latitude glaciers with particular consideration of the Peruvian Cordillera Blanca. Geograf. Ann., 81A(4), 643-651.

Kaser, G., Hastenrath, S., Ames, A., 1996. Mass balance profiles on Tropical glaciers. Z. Gletscherkd. Glazialgeol. 32, 75-81.

Kaser, G., 2002a: Glacier mass balance and climate in the South American Andes. In: Casassa, G., Sepulveda, F.V., Sinclair, R.M., (Eds.): The Patagonian Icefields,89-100.

Kaser, G., Osmaston, H., 2002b: Tropical Glaciers. Cambridge University Press,Cambridge.

Valladares, M. (2012). Estimación fotogramétrica del cambio producido en los páramos y glaciares del Volcán Antisana durante los últimos 50 años: desde 1956 hasta el 2009. Recuperado de www.mappinginteractivo.com. 